\title{
Searching for sub-stellar companions among nearby white dwarfs, through common proper motion
}

\author{
M. Radiszcz ${ }^{1,2}$ and R. A. Méndez ${ }^{1,3}$ \\ ${ }^{1}$ Departamento de Astronomia, Universidad de Chile, Casilla 36-D, Santiago, Chile \\ 2 email: madis@das.uchile.cl \\ 3 email: rmendez@das.uchile.cl
}

\begin{abstract}
The purpose of this work is to do a systematic, deep search for sub-stellar objects orbiting nearby white dwarfs (WDs). The scientific interest spans testing specific predictions of models of common envelope phase, as well as providing constraints to planetary system evolution in advanced stages of its parent star. Additionally, we seek to explore the hypothesis about the origin of metal lines in hydrogen WDs, produced by the accretion of tidal disturbed asteroidal or cometary material. This could be linked to the presence of a planetary object. Here, we show preliminary results of our near-infrared astrometric project.
\end{abstract}

Keywords. astrometry, binaries: close, stars: low-mass, brown dwarfs, stars: white dwarfs

\section{Sample selection and observations}

We select our targets from the most complete known sample of WDs within $20 \mathrm{pc}$ of the Sun, compiled by Holberg et al. (2002). Revisions and additional objects have been added (Kawka et al. 2004;2006). From WDs with $-80^{\circ}<\delta<20^{\circ}$, we select those WDs with young cooling + main sequence ages $(<3 \mathrm{Gyr})$, metal lines detected at their atmospheres, and with a possible near infrared excess. Targets in this sample have high proper motion $(>0.1$ arcsec) , so it is possible to confirm the sub-stellar companion candidates by looking for common proper motion pairs. We have obtained first epoch observations using VLT+NACO in Paranal Observatory in the J-band for 28 WDs. The frames obtained have a high-resolution (FWHM 0.1 arc-sec) go very deep ( $J_{\text {limit }} \sim 23.5-24 \mathrm{mag}$ ). We take advantage of the adaptive optical system by using the WD, which is brighter in the V-band, to correct the observations in the near infrared. We obtained high contrast and resolution diffraction-limited images at J-band, where cooler objects are more prominent. Nevertheless, the small FoV $\left(\sim 28 \times 28 \operatorname{arcsec}^{2}\right)$ does not allow us to explore larger projected separations. Therefore, to achieve a more extended FoV $\left(2 \times 2 \operatorname{arcmin}^{2}\right)$ , we have carried out additional observations with PANIC+Baade at Las Campanas Observatory. We obtained first epoch observation for $38 \mathrm{WDs}$, reaching a $5 \sigma$ detection limit at $21<J<22 \mathrm{mag}\left(\sim 5-10 M_{J u p}\right.$ orbiting a WD).

\section{Data analysis and results}

For PANIC data reduction, we use the IRAF pipeline gopanic, with very good results. We check for any signs of optical distortion that may have been left uncorrected. For NACO data reduction, we use the pipeline jitter provided by ECLIPSE. We find a very little "pincushion" distortion, but it is not significant enough $(<0.05$ pix for a typical 7 arc-sec of offset) to introduce problems in the reduced images and astrometric solutions. 
From 38 first epoch objects observed with PANIC, only 11 have at least a 1 year baseline for second epoch observations. These objects do not show common proper motion companions. For most of the objects, a 1 year of baseline is enough to detect common proper motion over $3 \sigma$ dispersion from the fainter objects detected on the frames (fainter objects exhibit larger residual RMS).

For the NACO frames, we have second epoch observations for 9 promising targets of which the data for 8 objects have been reduced. Some of these objects have faint candidates around 1 arc-sec from the WD. To properly study objects closer than 1 arcsec, it is necessary to execute a good PSF subtraction. IRAF DAOPHOT PSF models was not completely satisfactory. The best result could be obtained using a look-up table PSF model, based on a very similar WD (ideally the same WD without close companion) observed with the same instrumental configuration. Residuals after the PSF subtraction could be either real objects, optical effects or simply a PSF fit which does not exactly reproduce the central object. To improve the PSF subtraction, we observed the second epoch objects on two different rotation angles for the FoV. In this way we were able to discard some false detections produced by the NACO optical system (see a PSF subtracted image in Fig. 1-right). Despite the poor PSF subtraction within 1 arc-sec from the WD, it was possible to detect objects with a contrast of $\triangle J \sim 10$ mag, located just $\sim 1$ arc-sec off the target. Figure 1-left show a possible common proper motion companion, but it is not clear yet whether this object is real or not. Careful analysis of PSF substraction is necessary to confirm this detection. Until now, we have not had a confirmed detection of massive extra-giant planets among WDs. But a more detailed and complete analysis is needed to get a definitive detection.
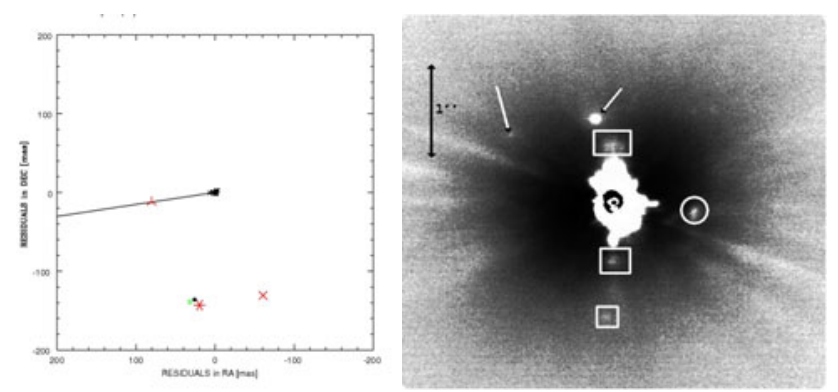

Figure 1. Left-Residuals of the position of stars between two frames from NACO WD residuals are plotted with the filled circle and consistent with the prediction (asterisk symbol) from proper motion (cross) and parallax (symbol over the line). A close object to the WD with a similar residual is marked with a open crossed square. To confirm common proper motion, a better analysis is necessary to discard a ghost image from the WD. Right-Residuals from one of our targets after of the PSF subtraction. Some residuals correspond to real objects (arrows); while others are due to optical effects or imperfect fitting of the PSF model (circles, squares).

\section{References}

Debes, J. H. \& Sigurdsson, S. 2002, ApJ, 572, 556

Livio, M. \& Soker, N. 1984, MNRAS 208, 763

Holberg, J. B. , Oswalt, T. D., \& Sion, E. M. 2002, ApJ 571, 512

Kawka, A. \& Vennes, S. 2006, ApJ 643, 402

Kawka, A., Vennes, S., \& Thorstensen, J. R. 2004, AJ 127, 1711

Willes, A. J. \& Wu, K., 2005, A\&ऽA 432, 1091 